\title{
Enhancing piezoelectricity through polarization-strain coupling in ferroelectric superlattices
}

\author{
Valentino R. Cooper 1 , * and Karin M. Rabe ${ }^{2}$ \\ ${ }^{1}$ Materials Science and Technology Division, Oak Ridge National Laboratory \\ Oak Ridge, Tennessee 37831-6114, USA \\ ${ }^{2}$ Department of Physics and Astronomy, Rutgers University \\ 136 Frelinghuysen Rd, Piscataway, New Jersey 08854-8019, USA
}

(Dated: July 2, 2018)

\begin{abstract}
Short period ferroelectric/ferroelectric $\mathrm{BaTiO}_{3}(\mathrm{BTO}) / \mathrm{PbTiO}_{3}(\mathrm{PTO})$ superlattices are studied using density functional theory. Contrary to the trends in paraelectric/ferroelectric superlattices the polarization remains nearly constant for PTO concentrations below 50\%. In addition, a significant decrease in the $c / a$ ratio below the PTO values were observed. Using a superlattice effective Hamiltonian we predict an enhancement in the $d_{33}$ piezoelectric coefficient peaking at $\sim 75 \%$ PTO concentration due to the different polarization-strain coupling in PTO and BTO layers. Further analysis reveals that these trends are bulk properties which are a consequence of the reduced $P$ brought about by the polarization saturation in the BTO layers.
\end{abstract}

PACS numbers: 77.65.-j, 77.84.-s, 68.65.Cd, 31.15.A-

Keywords: Ferroelectric superlattices, piezoelectric coefficients, first principles, $\mathrm{BaTiO}_{3}, \mathrm{PbTiO}_{3}$

Perovskite superlattices present a new paradigm for engineering ferroelectrics and piezoelectrics for modern device applications. Epitaxial strains, resulting from lattice mismatches, and induced changes in polarization, resulting from electrostatic considerations, can have significant effects on the macroscopic properties of these artificial structures. 1, 2] Superlattices combining a paraelectric (PE), such as $\mathrm{SrTiO}_{3}(\mathrm{STO})$, with a ferroelectric (FE), such as $\mathrm{PbTiO}_{3}$ (PTO) or $\mathrm{BaTiO}_{3}$ (BTO) have been well studied both by experiment and theory. [3, 4, 5, [6, 7] Their main features can be modeled by considering them as layers of strained bulk-like material with appropriate electrostatic boundary conditions [3]; in some systems, interface effects have also been shown to be important in the limit of ultrathin layers []. $]$. In this paper, we extend this first-principles modeling approach to superlattices combining two ferroelectrics, which we expect to exhibit new features arising from the interplay of the different bulk spontaneous polarizations and dielectric and piezoelectric coefficients of the two constituents.

We perform first-principles calculations, using density functional theory (DFT) [8, 9], for PTO/BTO superlattices with $m=1-3$ and $n=1-3$ layers of PTO and BTO, respectively. All calculations used projector augmented wave (PAW) potentials [10, 11] with the Vienna Ab initio Simulation Package (VASP v4.6.26)) 12], with the local density approximation for the exchange correlation functional. A $700 \mathrm{eV}(22 \mathrm{Ha})$ cutoff and a $6 \times 6 \times l \mathrm{k}-$ point mesh were used (where $l=4$ for periods 2 and 3 superlattices, 2 for periods 4 and 5 and 1 for period 6). For BTO in the tetragonal $P 4 \mathrm{~mm}$ 5-atom unit cell structure, the computed lattice constants are $\mathrm{a}=3.947 \AA$ and $\mathrm{c}=3.996 \AA$. The tetragonal PTO lattice constants were computed as $\mathrm{a}=3.867 \AA$ and $\mathrm{c}=4.033 \AA$ [experiment: $\mathrm{a}=3.904 \AA$ and $\mathrm{c}=4.152 \AA]$ [. 13 ] This agreement is

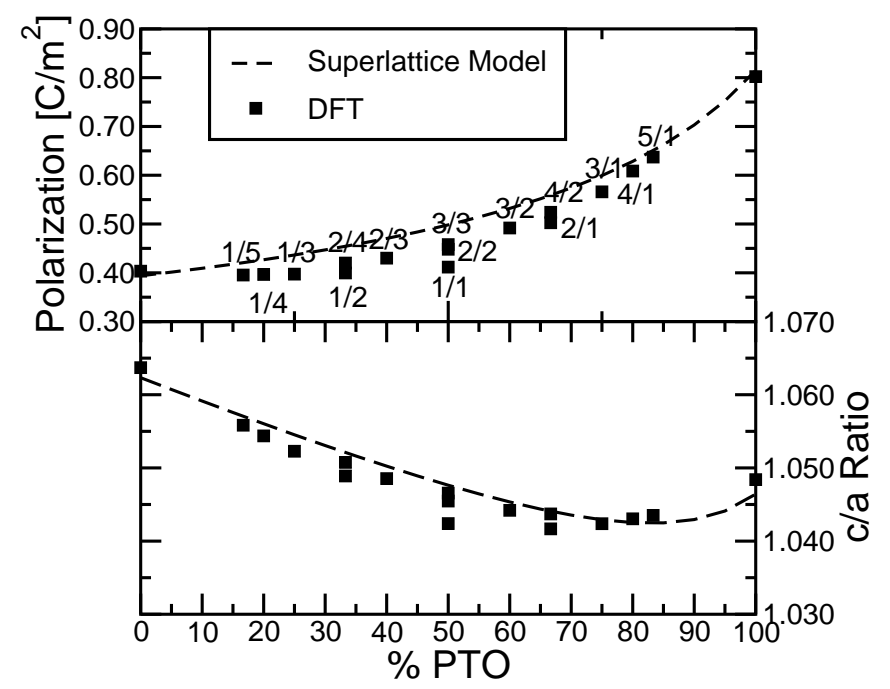

FIG. 1: Polarization along the $z$ axis (top) and $c / a$ ratio (bottom) as a function of \% PTO for BTO/PTO superlattices with varying superlattice periods. Solid squares represent DFT calculations and the dashed lines are the values obtained using the superlattice model (see Eqs. 1 and 3). Numerical model parameters can be found in Table —.

typical of LDA calculations for ferroelectric perovskites. In all superlattice calculations the in-plane lattice constant was constrained to that of the theoretical value for an STO substrate $(3.863 \AA)$ while the $c$ lattice vectors were optimized within the $P 4 \mathrm{~mm}$ space group with 1x1 in-plane periodicity. Additional calculations for a doubled $\sqrt{ } 2 \times \sqrt{ } 2$ in-plane unit cell for $m=n=1$ show no instability to octahedral tilts of the type discussed in Ref. 7. All ionic coordinates were fully relaxed until the Hellman-Feynman forces on the ions were less than $5 \mathrm{meV} / \AA$. Polarizations were computed using the Berry 
phase method [14]. For bulk PTO, constrained to the hypothetical STO substrate in-plane lattice constant of $3.863 \AA$, the out-of-plane lattice constant was $4.039 \AA$ and the polarization was $0.79 \mathrm{C} / \mathrm{m}^{2}$. The epitaxial constraints placed on the BTO layers result in a BTO $c / a$ ratio (1.063) much larger than that of the more polar PTO layers. The BTO polarization was $0.39 \mathrm{C} / \mathrm{m}^{2}$, substantially enhanced over the computed bulk value of $0.26 \mathrm{C} / \mathrm{m}^{2}$.

Figure 1 shows the dependence of computed polarization and $c / a$ lattice constant on the concentration of PTO and the superlattice period of short period PTO/BTO superlattices for the full set of structures with period $\leq$ 6 . Consistent with the fact that PTO has a greater polarization than BTO, higher concentrations of PTO give rise to larger $P$ of the superlattice. Similarly, decreasing the content of the larger $c / a$ ratio strained BTO reduces the average $c / a$ ratio of the superlattice. However, closer examination shows some unexpected features. First, the polarization of the superlattices remains almost constant for PTO concentrations below $50 \%$ while the $c / a$ ratio is below, rather than above, that of bulk PTO for a wide range of PTO concentrations ( $\sim 40 \%$ to $100 \%)$. In fact, the derivative of $c / a$ with respect to PTO concentration is positive near $100 \%$, rather than negative as would be expected from a simple linear interpolation.

To interpret our DFT data and to distinguish between interface effects and bulk properties, we constructed a parameterized energy expression for the superlattice following Refs. 15 and 16. The expression is a Taylor expansion around the cubic perovskite structure in terms of the six independent components $\eta_{i}$ of the strain tensor ( $i$ is a Voigt index, $i=1-6)$ and the three Cartesian soft mode amplitude components $u_{\alpha}(\alpha=x, y, z)$ parameterized from DFT calculations. Restricting to $P 4 \mathrm{~mm}$ symmetry, the effective stress-strain elastic enthalpy for a pure component ferroelectric under epitaxial strain (i.e. $\eta_{1}=\eta_{2}=\bar{\eta}$ ) with out-of-plane stress, $\sigma_{3}$, can be written as:

$$
\begin{array}{r}
G\left(\bar{\eta}, \eta_{3}, u_{z}, \sigma_{3}\right)=\frac{1}{2} B_{11}\left(2 \bar{\eta}^{2}+\eta_{3}^{2}\right)+B_{12}\left(\bar{\eta}^{2}+2 \eta_{3} \bar{\eta}\right)+ \\
\kappa u_{z}^{2}+\alpha u_{z}^{4}+\frac{1}{2} B_{1 x x} \eta_{3} u_{z}^{2}+B_{1 y y} \bar{\eta} u_{z}^{2}-\sigma_{3} \eta_{3}
\end{array}
$$

where $B_{11}$ and $B_{12}$ are related to the elastic constants of the crystal, $\kappa$ and $\alpha$ are two independent symmetryallowed fourth-order coefficients describing the cubic anisotropy and $B_{1 x x}$ and $B_{1 y y}$ are the phonon-strain coupling coefficients.

Figure 2 shows the polarization versus in-plane lattice constant for pure component BTO and PTO. The model parameters (Table【) were fit to DFT data for PTO with in-plane lattice constants within $\pm 1 \%$ of the STO in-plane lattice constant $3.863 \AA$. For BTO, the fitting range was extended to include the computed bulk tetrag-

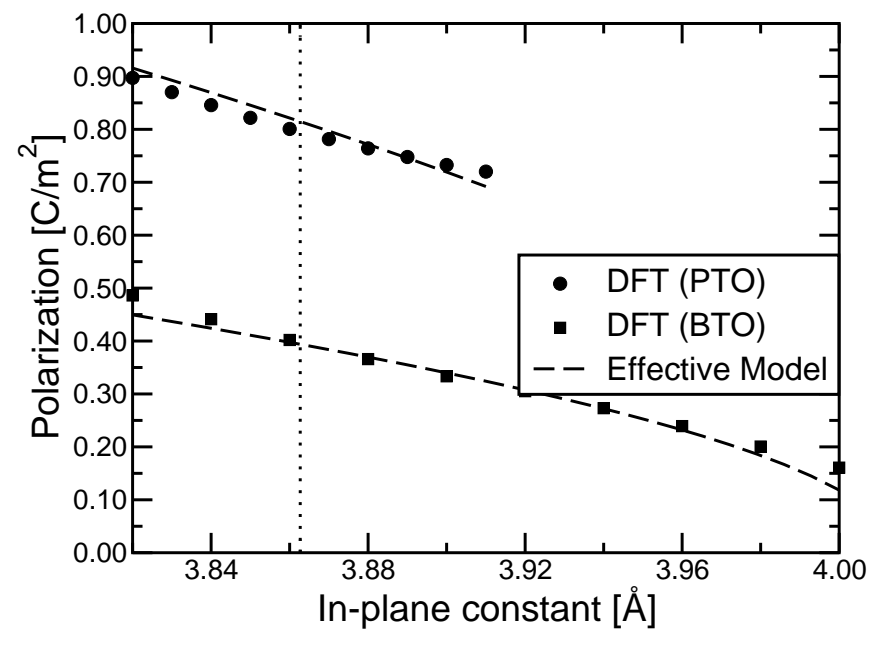

FIG. 2: Polarization along the $z$ axis as a function of inplane strain for bulk BTO and PTO. Solid circles and squares represent DFT calculations for PTO and BTO, respectively. Dashed lines are the corresponding values obtained using Eq. 1 with the parameters of Table [

onal lattice constant of $3.947 \AA$. The excellent agreement between the model and our DFT calculations is an indication of the quality of the model.

For a given $m_{\mathrm{PTO}} / n_{\mathrm{BTO}}$ superlattice we assume uniform soft mode amplitude $u_{z}$ within each constituent layer. Further, we impose the constraint that the corresponding polarizations of the two constituent layers $i=\mathrm{BTO}$, PTO,

$$
P_{z}^{i}=\frac{e}{\Omega^{i}} Z^{*, i} u_{z}^{i}
$$

are equal, where $e$ is the absolute value of the electron charge, $\Omega^{i}$ is the layer unit cell volume and $Z^{*, i}$ is the Born effective charge of the soft mode, so that polarization is uniform throughout the superlattice. Strictly speaking, it is the displacement field, $D=P+\varepsilon E$, which remains uniform throughout the superlattice. However, this uniform polarization approximation has previously been shown to be valid for short-period superlattices [6, 17] and is supported in the present case by unit-cell-layer polarization profiles computed using bulk Born effective charges (not shown).

The effective elastic enthalpy for the two-componentsuperlattice with $m_{\mathrm{PTO}}$ layers and $n_{\mathrm{BTO}}$ layers is then obtained as:

TABLE I: Energy expansion coefficients, Eq. 1. and soft mode Born effective charges, for PTO and BTO, in atomic units fit to the DFT results in Fig. 2, as described in the text.

\begin{tabular}{ccccccccc}
\hline \hline & $B_{11}$ & $B_{12}$ & $B_{1 x x}$ & $B_{1 y y}$ & $\kappa$ & $\alpha$ & $Z^{*}$ & $a_{\mathrm{o}}$ \\
\hline $\mathrm{BaTiO}_{3}$ & 5.13 & 3.05 & -1.00 & -0.100 & -0.007 & 0.14 & 9.94 & 7.48 \\
$\mathrm{PbTiO}_{3}$ & 4.69 & 1.15 & -0.705 & 0.207 & -0.0132 & 0.0364 & 9.40 & 7.385 \\
\hline \hline
\end{tabular}




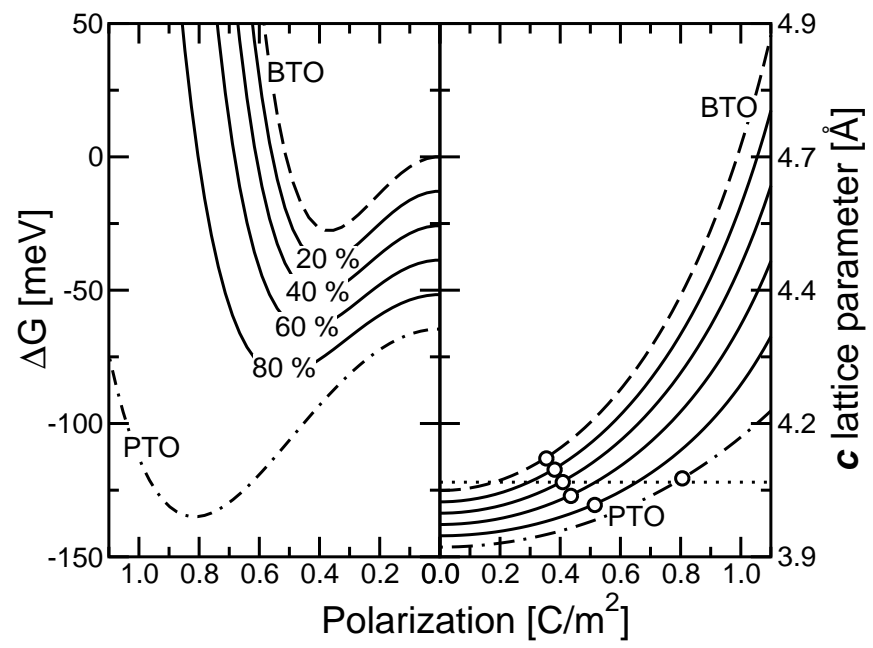

FIG. 3: Dependence of $d_{33}$ on PTO concentration for PTO/BTO superlattices. The dashed line represent the superlattice effective Hamiltonian at bulk STO in plane lattice constant of $3.863 \AA$ and the dotted line is are the same for an in-plane lattice constant of $3.90 \AA$. The solid squares are computed from DFT.

$$
G^{\text {Total }}=\frac{1}{(m+n)}\left[m G^{\mathrm{PTO}}+n G^{\mathrm{BTO}}\right] .
$$

The superlattice $P$ and $\eta_{3}$ are determined by minimizing Eq. 3 with respect to $\eta_{3}^{i}$ and $u_{z}^{i}$, imposing the uniform polarization approximation through the relation

$$
u_{z}^{\mathrm{BTO}}=\Gamma u_{z}^{\mathrm{PTO}}, \text { with } \Gamma=\frac{\Omega^{\mathrm{BTO}} Z^{*, \mathrm{PTO}}}{\Omega^{\mathrm{PTO}} Z^{*, \mathrm{BTO}}} .
$$

This model can also be used to compute the piezoelectric coefficient, $d_{33}$, as:

$$
\begin{aligned}
\frac{d P}{d \sigma_{3}}= & -\frac{m e Z^{*, P T O} B_{1 x x}^{P T O}}{2 \Omega^{P T O} u_{z}^{P T O}\left(2 B_{11}^{P T O} \alpha^{P T O}-\frac{1}{4}\left(B_{1 x x}^{P T O}\right)^{2}\right)} \\
& -\frac{m e Z^{*, B T O} B_{1 x x}^{B T O}}{2 \Omega^{B T O} u_{z}^{B T O}\left(2 B_{11}^{B T O} \alpha^{B T O}-\frac{1}{4}\left(B_{1 x x}^{B T O}\right)^{2}\right)} .
\end{aligned}
$$

Figure 3 shows the dependence of $d_{33}$ on composition as predicted from Eq. 5. The model is in good agreement with the DFT $d_{33}$ values for bulk BTO $(32 \mathrm{pC} / \mathrm{N})$, bulk PTO $(55 \mathrm{pC} / \mathrm{N})$ and the $3 \mathrm{PTO} / 1$ BTO superlattice $(58 \mathrm{pC} / \mathrm{N})$. Surprisingly, we find a $5 \%$ enhancement of the $d_{33}$ coefficient at $75 \% \mathrm{PTO}$ where the $c / a$ is a minimum.

The good agreement between the DFT results and the superlattice energy functional, shown in Fig. 1. suggests that the observed trends in $\mathrm{P}$ and $c / a$ are a consequence of bulk electrostatics and strain effects, and that interfaces do not play an essential role. The success of the model allows us to elucidate the origin of the nearly constant $P$ for low PTO concentration through an examination of the evolution of the FE potential energy well
(Fig. 4, left panel). A comparison of the FE potential energy wells of the epitaxially strained parent compounds shows that BTO has a much stiffer FE well with a minimum at a $P$ of less than half of PTO. For polarizations much greater than $0.39 \mathrm{C} / \mathrm{m}^{2}$ the effective enthalpy of BTO sharply increases, while PTO has a much gentler dependence. Here the $P$ in the BTO layers is saturated, requiring larger electric fields to further polarize them. Similar polarization saturation effects have been observed in compressively strained PZT and PTO [18]. In a superlattice, this translates into the need for a higher percentage of the more polar PTO in order to increase the macroscopic polarization of the superlattice. This fact is borne out in the evolution of the FE wells with \% PTO. Even up to PTO concentrations of $80 \%$ the FE well still resembles that of BTO, severely limiting the total $P$ in the superlattice and accounting for the suppression in expected $P$ enhancement with increasing \% PTO.

This suppressed layer-by-layer polarization can be linked to the abnormal decrease of $c / a$ below that of the PTO parent compound (Fig. 4) as well as the enhancement in the $d_{33}$ coefficient. The model indicates that if $P$ were to increase linearly with increasing PTO concentration then the $c$ lattice parameter would have a corresponding linear decrease to the PTO values. However, as previously stated, the $P$ throughout the superlattices is severely suppressed due to the presence of the BTO layers. Since $P$ is coupled to the $c$ lattice parameter this results in a drastic reduction in the average $c$ lattice parameter (see Fig. 4). This BTO effect is only overcome at extremely high concentrations of PTO, where the electric field generated by the PTO layers are capable of counteracting the BTO potential energy well constraints. Furthermore, BTO has a much steeper depen-

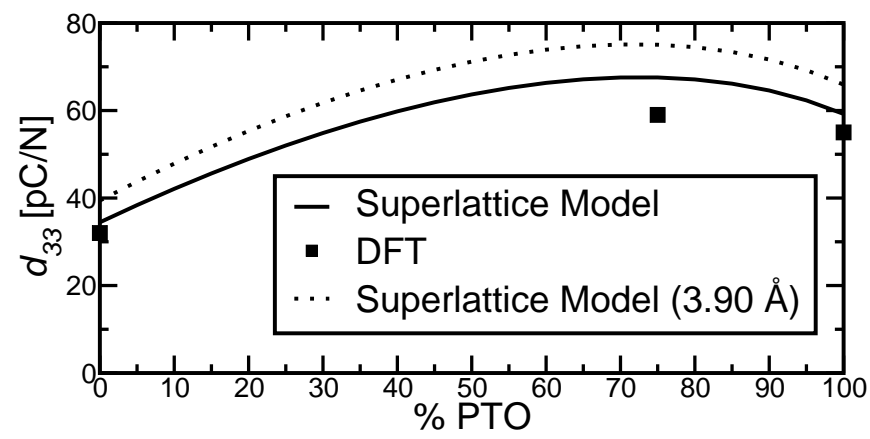

FIG. 4: Effective enthalpy (left) and $c$ lattice parameter (right) as a function of polarization for various compositions of PTO/BTO superlattices obtained from the superlattice energy functional. Dashed lines represent pure BTO, dasheddotted lines are for pure PTO and solid lines indicate the ferroelectric wells for the superlattices at $20 \%$ PTO intervals. Open circles mark the c lattice parameter for each composition at the predicted $P$. The dotted line in the right figure indicates $c$ for epitaxially-strained tetragonal PTO. Energies are relative to epitaxially strained bulk BTO. 
dence of $c$ on $P$ than PTO, i.e. the $c$ lattice parameter increases more with increasing $P$ in the BTO layers than in the PTO layers. As such, it is the balance between the stronger polarization-strain coupling in the BTO layers and the amount of PTO present to pole these layers which give rise to the peak in $d_{33}$ at $\sim 75 \%$ PTO concentration. These results suggest that it may be possible to further enhance $d_{33}$ by reducing the saturation in the BTO layers either by decreasing the in-plane compressive strain (see Fig. 4, dotted line: $a=3.90 \AA$ ) or through other factors such as intermixing at the interface between PTO and BTO layers. 6]

In conclusion, we have used density functional theory to explore the dependence of $P$ and $c / a$ as a function of PTO concentration for short period PTO/BTO superlattices. Our extensive DFT calculations reveal two intriguing phenomena: nearly constant $P$ for PTO concentrations less than $50 \%$ and a dramatic decrease in $c / a$ to values below that of the smaller PTO. Using a superlattice effective Hamiltonian we demonstrate that these trends are a consequence of $P$ saturation in the BTO layers which, by limiting the magnitude of $P$, results in abnormally large decreases in the $c$ lattice parameter. The competition between the polarization saturation effects of BTO and the high polarization in the PTO layers results in a corresponding peak in the $d_{33}$ coefficients in the PTO/BTO superlattices, suggesting a new way of enhancing the piezoelectric properties of the superlattices; other material combinations may yet be found which show an even greater enhancement based on this mechanism. In addition, this model can be easily extended to multicomponent systems, different phases and to model strain effects on these phases; allowing for the easy exploration of materials properties.

We would like to thank David Vanderbilt and Scott Beckman for valuable discussions. This work was supported by ONR Grant N0014-00-1-0261. Part of this work was carried out at the Aspen Center for Physics. Work at ORNL was supported by DOE, Division of Ma- terials Sciences and Engineering.

Electronic address: coopervr@ornl.gov

[1] M. Dawber, K. M. Rabe, and J. F. Scott, Rev. Mod. Phys. 77, 1083 (2005).

[2] H. N. Lee, H. M. Christen, M. F. Chisholm, C. M. Rouleau, and D. H. Lowndes, Nature 433, 395 (2005).

[3] J. B. Neaton and K. M. Rabe, Appl. Phys. Lett. 82, 1586 (2003).

[4] K. Johnston, X. Huang, J. B. Neaton, and K. M. Rabe, Phys. Rev. B 71, 100103(R) (2005).

[5] M. Dawber, C. Lichtensteiger, M. Cantoni, M. Veithen, P. Ghosez, K. Johnston, K. M. Rabe, and J. M. Triscone, Phys. Rev. Lett. 95, 177601 (2005).

[6] V. R. Cooper, K. Johnston, and K. M. Rabe, Phys. Rev. B 76, 020103(R) (2007).

[7] E. Bousquet, M. Dawber, N. Stucki, C. Lichtensteiger, P. Hermet, S. Gariglio, J.-M. Triscone, and P. Ghosez, Nature 452, 732 (2008).

[8] P. Hohenberg and W. Kohn, Phys. Rev. 136, B864 (1964).

[9] W. Kohn and L. J. Sham, Phys. Rev. 140, A1133 (1965).

[10] P. E. Blöchl, Phys. Rev. B 41, 5414 (1990).

[11] G. Kresse and D. Joubert, Phys. Rev. B 59, 1758 (1999).

[12] G. Kresse and J. Furthmüller, Phys. Rev. B 54, 11169 (1996).

[13] K. Hellwege and A. M. Hellwege, eds., LandoltBörnstetin, vol. III (Springer-Verlag, Berlin, 1981).

[14] R. D. King-Smith and D. Vanderbilt, Phys. Rev. B 47, 1651 (1993).

[15] R. D. King-Smith and D. Vanderbilt, Phys. Rev. B 49, 5828 (1994).

[16] O. Diéguez, K. M. Rabe, and D. Vanderbilt, Phys. Rev. B 72, 144101 (2005).

[17] S. M. Nakhmanson, K. M. Rabe, and D. Vanderbilt, Appl. Phys. Lett. 87, 102906 (2005).

[18] H. N. Lee, S. M. Nakhmanson, M. F. Chisholm, H. M. Christen, K. M. Rabe, and D. Vanderbilt, Phys. Rev. Lett. 98, 217602 (2007). 